\title{
Intrauterine growth restriction impairs right ventricular response to hypoxia in adult male rats
}

\author{
Michael Keenaghan ${ }^{1,2}$, Lena Sun ${ }^{3,4}$, Aili Wang ${ }^{3}$, Eiichi Hyodo ${ }^{5}$, Sinichi Homma ${ }^{5}$ and Vadim S. Ten ${ }^{4}$
}

BACKGROUND: Intrauterine growth restriction (IUGR) predisposes to cardiovascular diseases in adulthood. The mechanisms of this phenomenon remain cryptic. We hypothesized that heart mitochondria in IUGR-born adult rats are more sensitive to acute hypoxia which translates into dysfunctional cardiac response to hypoxic stress.

METHODS: Adult IUGR-born male rats (the offspring of dams fed with calories-restricted diet during pregnancy) were exposed to acute hypoxic stress with echocardiographic assessment of cardiac function. In parallel, mitochondrial respiration in organelles isolated from left ventricle (LV) and right ventricle (RV) was tested in normoxic and anoxic conditions. The extent of post-anoxic inhibition of mitochondrial respiration and cardiac function was compared with controls, non-IUGR rats.

RESULTS: Compared with controls, in the IUGR rats hypoxia significantly reduced only RV contractility, evidenced by decreased fractional shortening, functional area of contraction, and tricuspid annular plane systolic excursion. In isolated mitochondria, anoxic challenge inhibited respiratory chain in both groups of rats. However, compared with controls, the extent of anoxic mitochondrial depression was significantly greater in IUGR-born rats, but only in the organelles isolated from RV.

CONCLUSIONS: In adult IUGR-born rats, mitochondria from $\mathrm{RV}$ are hypersensitive to oxygen deprivation and this translates into maladaptive RV cardiac response to acute hypoxia.

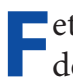
etal programming, first proposed by Hales and Barker (1), describes the developmental origins of health and disease $(2,3)$. Fetal stresses and environmental factors, including under- or overnutrition, anemia, exposure to cocaine, and maternal smoking, induce changes in genes, proteins, or metabolic pathways implicated in remodeling of the hypothalamicpituitary-adrenal axis $(4,5)$, obesity, and in increased risk of diseases of the cardiovascular system (6).

Cardiovascular changes associated with intrauterine growth restriction (IUGR) include centralization of the arterial circulation (7), increases in fetal afterload, cardiac output, remodeling of the heart, and changes in arterial compliance (8). Maternal under- and overnutrition is known to influence cardiac function $(7,8)$ and total cardiac mitochondrial activity in their juvenile offspring (9). Newborn rat offspring born to protein-restricted dams have smaller hearts with fewer cardiomyocytes and are found to have cardiac remodeling and cardiac output changes (increased afterload and reduced arterial compliance) later in their life $(8,10)$. IUGR rats are found to have impaired adenosine triphosphate production (11) and oxidative phosphorylation in cardiomyocytes as well as cells from other major organs (12-15). Impaired oxidative phosphorylation has been linked to cardiomyocyte apoptosis, poor cardiac function, and dysfunctional mitochondrial Complex I and II, evident prior to the onset of cardiomyocyte apoptosis during transition from compensated left ventricular hypertrophy to failure (16).

Chamber-specific differences in mitochondrial respiration have been shown to occur in response to stress. Mitochondria from both LV and RV show evidence of morphological and functional phosphorylation defects in response to chronic hypoxia, but the onset of these events in RV is delayed compared with LV (17). In contrast, there is a selective decrease in Complex I activity in RV compared with LV in rats with right heart failure secondary to pulmonary arterial hypertension associated with hypoxia (18). Theoretically, in IUGR-born mature subjects, hypoxic stress (sleep apnea, pneumonia, acute respiratory distress syndrome, etc.) may manifest with exacerbated RV dysfunction compared with non-IUGR born, normal population.

Our study tests the hypothesis that compared with nonIUGR control counterparts, in IUGR-born adult male rats, acute hypoxic stress induces significantly greater RV dysfunction, and this is associated with selectively greater inhibition of $\mathrm{RV}$ cardiac mitochondrial respiratory chain.

\section{RESULTS}

\section{IUGR Model}

Before nutrient restriction, dam's weights were similar in both groups. As expected, nutrient restriction significantly reduced weight gain throughout the treatment period (Figure 1a). No difference in litter size was detected (Control $12.7 \pm 0.38$; IUGR $13.2 \pm 0.36$ pups). In IUGR offspring, body and heart weights at the initial day of life were significantly poorer compared with

'Department of Pediatrics, Kings County Hospital Center, Brooklyn, New York; ${ }^{2}$ Department of Pediatrics, St. Georges University, Grenada, West Indies; ${ }^{3}$ Department of Anesthesiology, Columbia University, New York, New York; ${ }^{4}$ Department of Pediatrics, Columbia University, New York, New York; ${ }^{5}$ Department of Medicine, Columbia University, New York, New York. Correspondence: Vadim S.Ten (vt82@cumc.columbia.edu)

Received 27 January 2016; accepted 23 April 2016; advance online publication 24 August 2016. doi:10.1038/pr.2016.124 


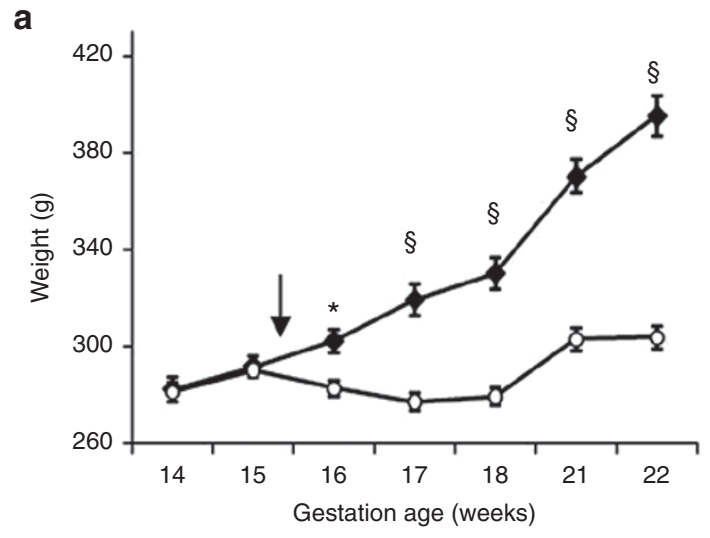

b

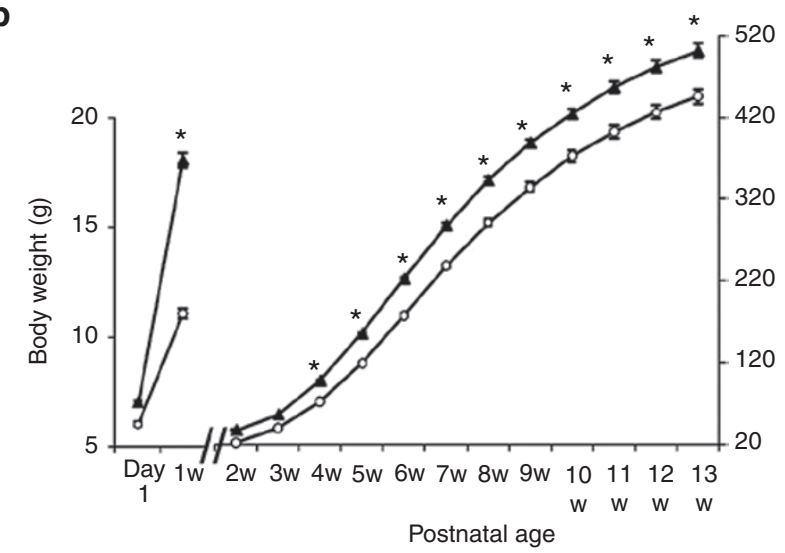

C

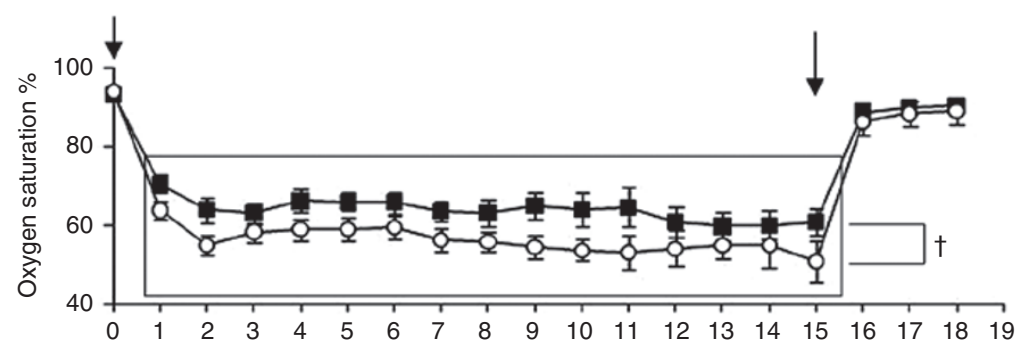

d

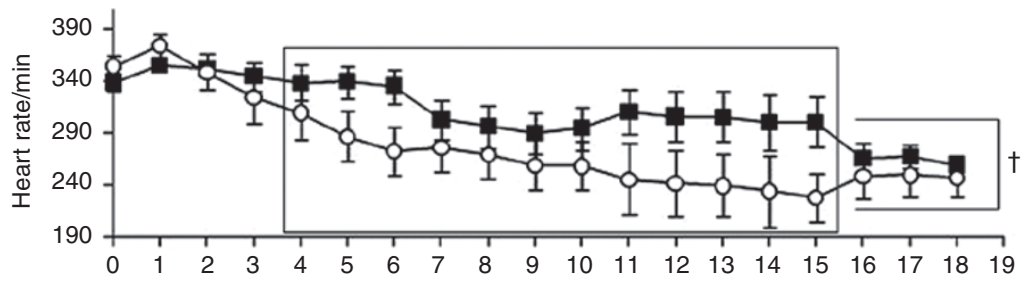

e

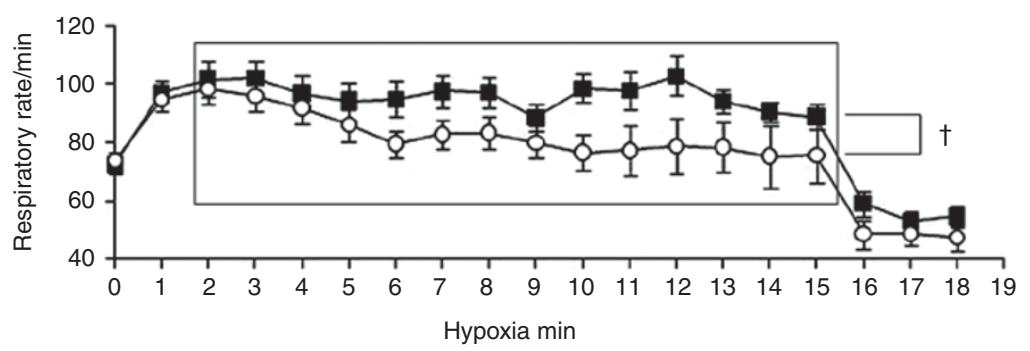

Figure 1. Physiological parameters. (a) Body weight changes in pregnant rats. Controls, black circles, $n=26$ and IUGR, open circles, $n=26 .{ }^{*} P<0.05,{ }^{* *} P$ $<0.001$, compared with IUGR rats. Arrow indicates start of feeding restriction. (b) Postnatal weight changes. Controls, black circles, $n=72$ and IUGR, open circles, $n=71 .{ }^{*} P<0.05,{ }^{* *} P<0.0001$, compared with IUGR group. (c-e) Changes in oxygen saturation $\left(\mathrm{SaO}_{2}\right)$, heart rates, and respiratory rates in control $(n=10)$ and IUGR $(n=9)$ offspring during hypoxia. Data demonstrating significant difference between groups are outlined. Arrows indicate start and stop of hypoxia. Controls, black circles; IUGR, open circles. ${ }^{*} P<0.0001$. IUGR, intrauterine growth restriction; NR, nutritional restriction.

controls (Figure $1 \mathrm{~b}$ and Table 1). However, the ratio of heart weight to body weight was similar in both groups (Table 1). Maternal nutrient restriction significantly affected offspring body weights beyond neonatal and weaning age. At all tested ages, body weights in IUGR offspring were significantly lower compared with controls (Figure 1b).

\section{Hypoxia and Echocardiography}

Control and IUGR rats exhibited comparable baseline (prehypoxic) $\mathrm{SaO}_{2}$, heart rate (HR) and respiratory rate (RR)
(Figure 1c-e), and cardio-functional outcomes obtained by echocardiography (ECHO). Exposure to hypoxia caused a significant decrease in $\mathrm{SaO}_{2}$, associated with significant increase in RRs compared with baseline values in both groups of rats (Figure 1c,e). Oxygen desaturation occurred more rapidly in IUGR rats compared with controls, as indicated by a steeper slope of decline in $\mathrm{SaO}_{2}$ with a lower nadir. HRs in IUGR and control rats were comparable at baseline and at $1 \mathrm{~min}$ of hypoxia, progressively declining to a greater extent in IUGR animals during hypoxia compared with controls (Figure 1d). 
Table 1. Maternal and litter characteristics

\begin{tabular}{lcc}
\hline & Control & IUGR \\
\hline Maternal anthropometrics & & \\
Total number of pregnant rats & 26 & 26 \\
Weight at gestation date $15(\mathrm{~g})$ & $291.9 \pm 4.4$ & $290.4 \pm 3.1$ \\
Weight at gestation date 22 (g) & $395.5 \pm 8.4$ & $303.8 \pm 4.5^{* *}$ \\
Litter size (number of pups) & $12.7 \pm 0.38$ & $13.2 \pm 0.36$ \\
Pup anthropometrics & & \\
Body weight (g) & $7.0 \pm 0.1(n=188)$ & $6.1 \pm 0.1(n=174)^{*}$ \\
Heart weight (mg) & $31.9 \pm 0.58(n=60)$ & $27.2 \pm 0.5(n=64)^{*}$ \\
Heart/body weight ratio & $0.46 \pm 0.01(n=60)$ & $0.46 \pm 0.01(n=60)$ \\
\hline IUGR, intrauterine growth restriction. & & \\
Values are mean \pm SE. ${ }^{*} P 0.05 * * * 0.001$. &
\end{tabular}

a
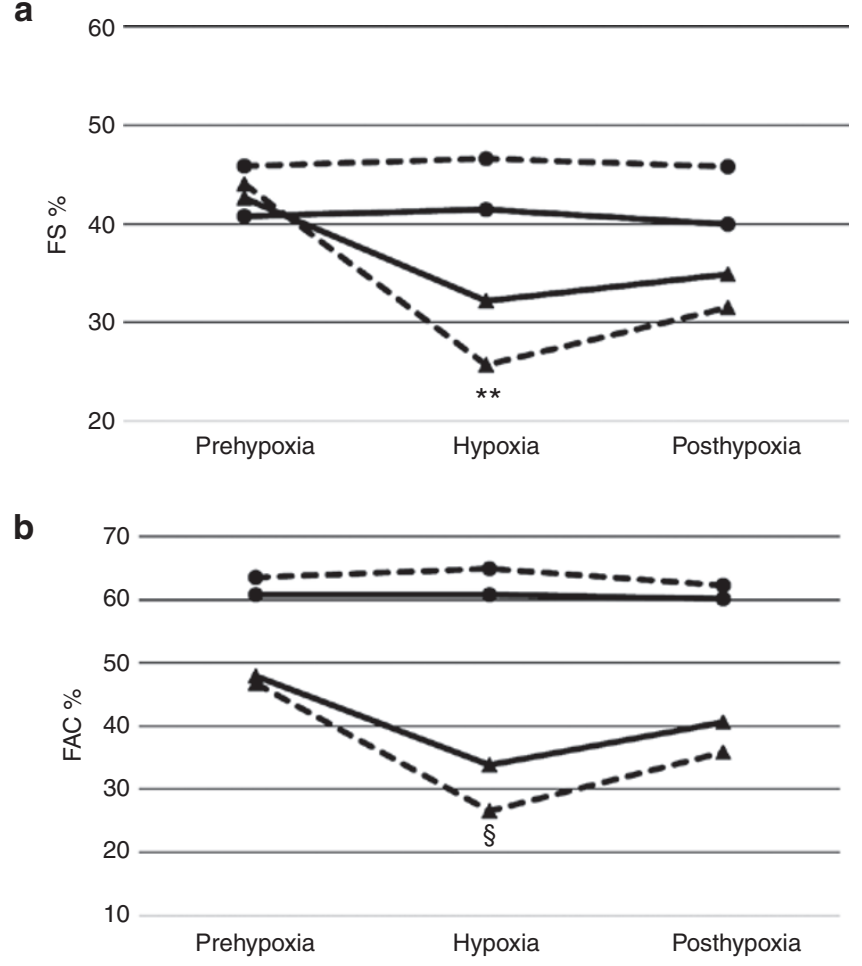

C

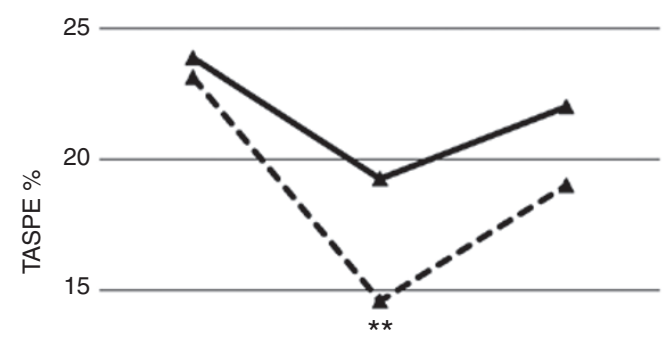

10

Prehypoxia Hypoxia Posthypoxia

Figure 2. Echocardiographic indices in Control and IUGR rats, prior to-, during and after hypoxia. (a) Fractional shortening \%, (b) fractional area change \%, and (c) tricuspid annular plane systolic excursion. Circle with solid line is Control LV; circle with dotted line is IUGR LV; triangle with solid line Control RV and triangle with dotted line is IUGR RV. ${ }^{*} P<0.01,{ }^{* *} P<$ 0.001 compared with corresponding values in controls. IUGR, intrauterine growth restriction; $\mathrm{LV}$, left ventricle; $\mathrm{RV}$, right ventricle.
RR in both groups increased at the same rate for the initial 2 min and then the rate of RR decline was significantly faster in the IUGR group (Figure 1e). Upon reoxygenation with room air, $\mathrm{SaO}_{2}$ normalized within $2 \mathrm{~min}$ in both groups. Post-hypoxic HR and RR did not differ between control and IUGR rats.

Results of echocardiographic exam are shown in Figure 2. Pre-hypoxic values of LV and RV functions did not differ between groups. In response to hypoxic challenge, LV systolic and diastolic dimensions and areas did not change in both groups of rats. However, RV systolic and diastolic areas significantly enlarged in control rats (pre-hypoxia, $49.6 \pm 1.9$ vs. hypoxia, 74.8 $\pm 1.6 ; P<0.001$ ) and in their IUGR counterparts (pre-hypoxia, $61.1 \pm 1.7$ vs. hypoxia, $83.2 \pm 1.5 ; P<0.001$ ). Importantly, in IUGR rats RV systolic function assessed by fractional shortening, fractional area change, and tricuspid annular plane systolic excursion decreased to a significantly greater degree compared with controls (Figure $2 \mathbf{a}-\mathbf{c}$ ). No significant differences in LV systolic function were detected in response to hypoxia in both groups of rats (Figure 2a,b).

\section{Mitochondrial Respiration}

The baseline, normoxic, state 4 (resting respiration) and adenosine diphosphate (ADP)-stimulated, state 3, and uncoupled, 2,4-dinitrophenol (DNP)-induced (DNP1), respirations were comparable in RV and LV between control and IUGR rats (Figure 3a,b). Following anoxic challenge, the DNP2 (uncoupled respiration measured after anoxia) rate in both groups of rats in both ventricles significantly decreased compared with that in normoxia (Figure 3-c-f). The extent of this anoxic depression of mitochondrial respiratory chain differed depending upon substrates: Organelles fueled with succinateglutamate (complex-II (C-II) dependent substrate) were less affected with anoxia, compared with the same mitochondria fueled with malate-glutamate (complex-I (C-1) dependent substrate) (Figure 3b,d). In control rats, mitochondria isolated from both LV and RV were depressed to a similar extent following anoxia. However, in IUGR rats, cardiac mitochondria isolated only from the RV demonstrated significantly poorer DNP2 $\mathrm{O}_{2}$ consumption rate compared with post-anoxic RV mitochondria from control rats (Figure 3f). This poorer mitochondrial respiratory chain function was detected only when RV mitochondria were supported with C-I dependent substrates (malate-glutamate), but not C-II dependent succinate (Figure 3d,f).

Electron microscopy exam of RV isolated mitochondrial pellets revealed intact and well-preserved organelles in both control and IUGR rats (Figure 4a,c). Anoxic challenge markedly changed mitochondrial morphology, causing swelling of organelles and partial loss of matrix cristae in both groups of rats (Figure $4 b, d$ ).

\section{DISCUSSION}

This study is the first to demonstrate a significant exacerbation of $\mathrm{RV}$ systolic dysfunction in response to acute hypoxic stress in adult rats born with IUGR. In IUGR-born rats only RV function, but not LV function, was depressed in response to acute hypoxic stress and during recovery. Mechanistically, this 
a
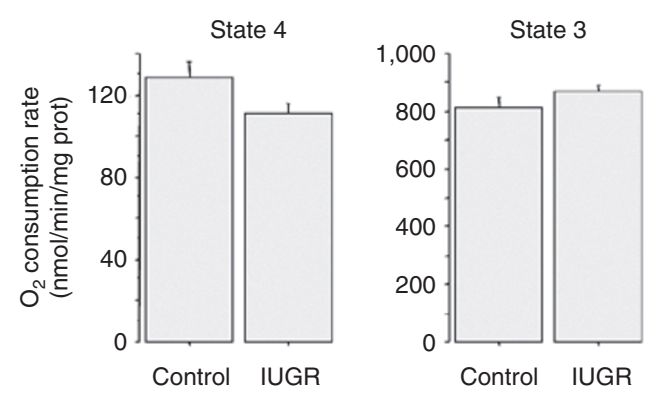

C

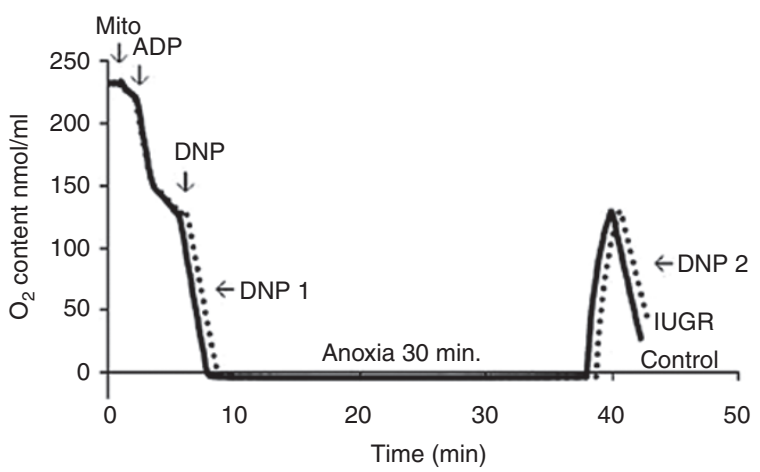

e

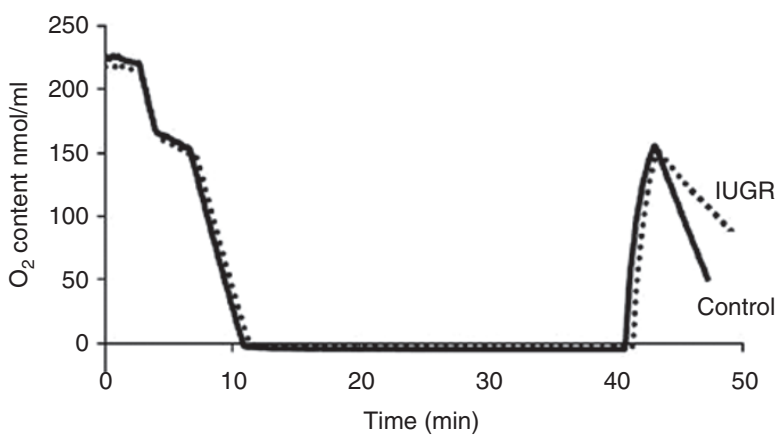

b

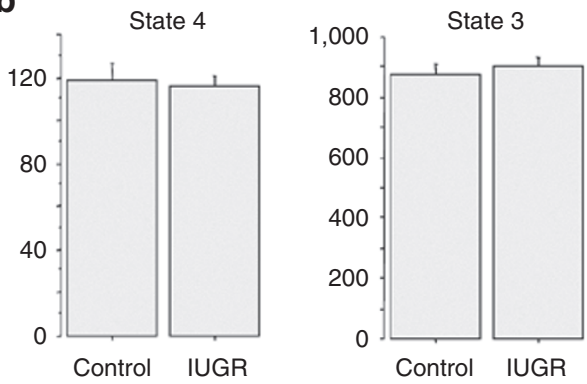

d

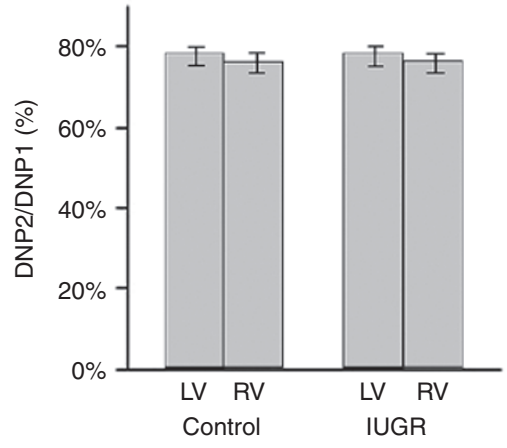

f

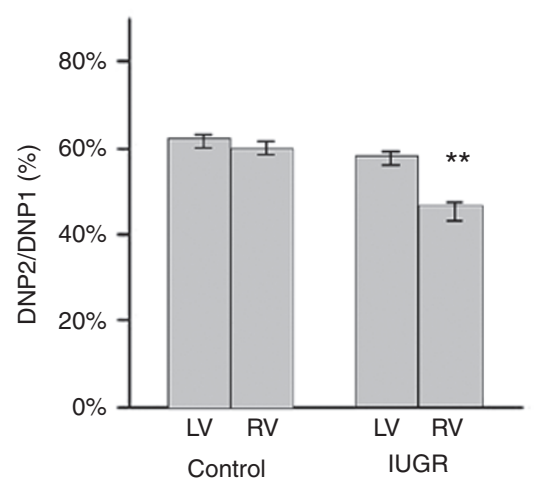

Figure 3. Mitochondrial respiration before and after anoxia. Baseline state 4 (a) and state 3 (b) RV mitochondrial $\mathrm{O}_{2}$ consumption rates. Substrates, malate-glutamate ( $n=11$, IUGR and 10, Control). (c) Representative tracing of RV mitochondrial respiration supported with succinate-glutamate; control (bold line) and IUGR (dotted line). (d) The ratios of post-anoxic to pre-anoxic mitochondrial maximal (uncoupled) respiration (DNP2/DNP1 in \%) in organelles isolated from LV or RV in control $(n=4)$ or IUGR rats $(n=4)$. Substrate, succinate-glutamate. (e) Representative tracing of RV mitochondrial respiration supported with malate-glutamate; control (bold line) and IUGR (dotted line). (f) The ratio of post-anoxic to pre-anoxic mitochondrial maximal (uncoupled) respiration (DNP2/DNP1 in \%) in organelles isolated from LV or RV in control $(n=10)$ or IUGR rats $(n=11)$. Mitochondria were fueled with malate-glutamate. Arrows: MITO, addition of mitochondria; ADP, addition of ADP, DNP, addition of DNP. **P $<0.001$ compared with mitochondria from control RV. ADP, adenosine diphosphate; DNP, 2,4-dinitrophenol; IUGR, intrauterine growth restriction; LV, left ventricle; RV, right ventricle.

IUGR-associated exacerbation of RV dysfunction was linked to a significantly greater sensitivity of RV mitochondria to oxygen deprivation compared with that in control rats.

There are numerous IUGR models that have been used to investigate fetal programming of adult diseases (19-21), including the model of caloric restriction, that we have chosen for this study. Our anthropometric data are consistent with results of other reports on this model (10,20-23). Body weights in IUGR rats remained significantly lower than in controls at all studied ages, although an accelerated (catch-up) growth rate in IUGR groups generally occurs around 2-3 wk of life $(24,25)$. However, this usually does not occur, if maternal protein/caloric restriction is maintained after birth and during the preweaning period, as it was in our model $(21,26,27)$. The proportion of heart weight to body weight in two groups was not statistically different, and this is also consistent with previous observations $(10,20,21)$. Based on studies $(10,20-23)$, one may conclude that the heavier rats have bigger hearts and vice versa. This suggests that postnatal heart size is a function of overall somatic growth. Functional implication of fetal IUGR on cardiac performance was assessed during acute hypoxic challenge, the most common life-threatening stress in humans. Here we show that in response to acute hypoxemia, RV contractility was significantly reduced in both groups of rats. However, the extent of this RV systolic dysfunction (evidenced by a shorter tricuspid annular plane systolic excursion distance 

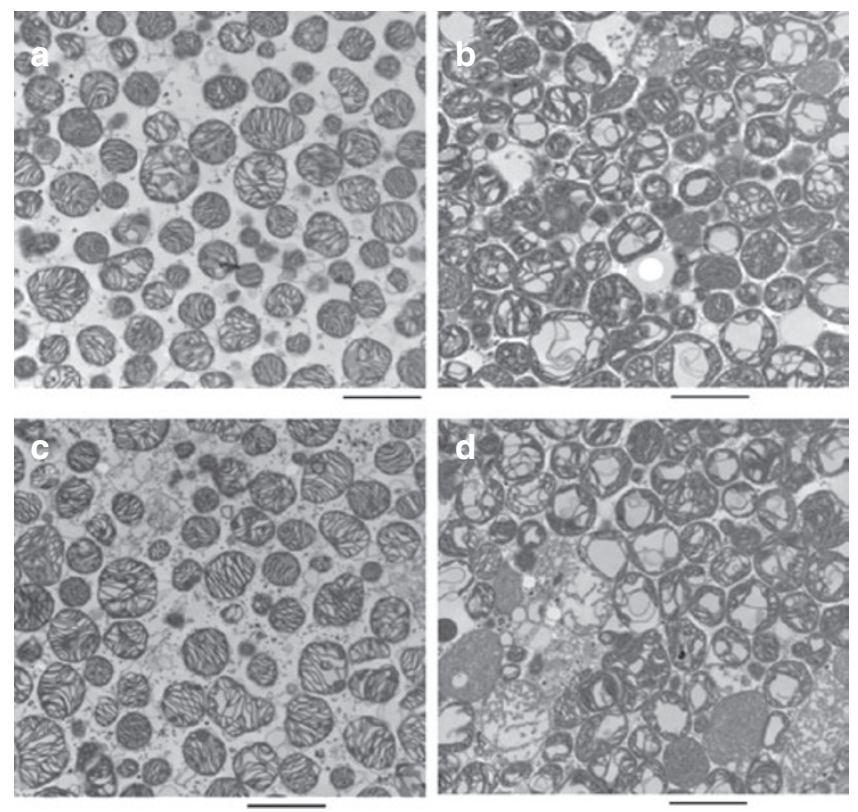

Figure 4. Electron microscopy of RV mitochondria. Electron microscopy of mitochondrial pellets isolated from RV of control before (a) and after anoxic challenge (b) and IUGR rats before (c) and after anoxic challenge (d). Scale bar $=2 \mu \mathrm{m}$. IUGR, intrauterine growth restriction.

and decreased fractional area change) was significantly greater in IUGR animals compared with controls. This suggests that following fetal IUGR, the RV, even in mature subjects, is less tolerant to hypoxic depression. Thus, IUGR can be considered as a risk factor for more severe selective RV dysfunction during acute hypoxic stress.

It is possible that compared with controls, significant worsening of RV dysfunction in IUGR-born rats was secondary to a slightly greater extent of hypoxemia during hypoxic challenge (Figure 1c). Indeed, mechanistically, RV dysfunction during hypoxia has been explained by the hypoxic elevation in pulmonary vascular resistance, resulting in a greater RV afterload. In isolated hearts, however, without the effect of the lungs, hypoxemic perfusion also reduced RV contractility and glucose utilization, the event associated with inefficient $\mathrm{Na}^{+} / \mathrm{K}^{+}$-adenosine triphosphatase function (28). Our study revealed that in the IUGR-born rats mitochondria isolated only from RV are more sensitive to hypoxic inhibition compared with that in their control counterparts. Given that myocardial contractility directly depends upon availability of high-energy substrates, more severe inhibition of the mitochondrial respiratory chain during oxygen deprivation may serve as another plausible explanation for significantly exacerbated RV dysfunction in IUGR animals compared with their controls. By testing mitochondria with different substrates, we have found that RV organelles exhibited severe post-anoxic respiratory inhibition only when these organelles were supported with nicotinamide adenine dinucleotide (NAD)linked substrates, malate-glutamate which provide electron flux via C-I. In contrast, when the same mitochondria were fueled with succinate, no difference in post-anoxic (DNP2) respiration rates was detected between controls and IUGR rats. Because, succinate is Flavin adenine dinucleotide (FAD)linked substrate and supports electron flux via C-II, these data suggest that IUGR selectively affects sensitivity of C-I to oxygen deprivation. This highlights $\mathrm{C}$-I is a mechanistic target responsible for exacerbation of mitochondrial dysfunction in IUGR-born adult subjects during hypoxic stress.

C-I can undergo a conformational change from an active form, A-form, to a deactivated form, D-form, in response to acute hypoxia (29), hyperthermia (30), or increased $\mathrm{Ca}^{2+}$ concentration (31) or fatty acids (32). Galkin et al. have (29) hypothesized that the $\mathrm{D}$-form may be a protective mechanism against mitochondrial overproduction of reactive oxygen species during hypoxic event, and after reoxygenation, conformational return to A-form normalizes bioenergetics. Indeed, partial inhibition of C-I during ischemia protected cardiac and brain mitochondria from reperfusion injury (33-35). It is possible that in the IUGR rats RV mitochondria are more prone to this $\mathrm{D}$-form conformation compared with control animal. However, our in vivo data suggest that in IUGR-born rats this hypothetical conformational change in C-I is associated with exaggerated RV dysfunction, rather than with an adaptogenic event. It has been shown that IUGR affects $\mathrm{NAD}^{+} / \mathrm{NADH}$ ratio and reduces expression of adenine-nucleotide translocator 1, ATP-synthase, and NADH-ubiquinone oxidoreductase in skeletal muscles of juvenile IUGR-born rats (36). Based on these findings, authors concluded that IUGR negatively affects skeletal muscles mitochondrial function. Our data extend this observation by showing that exacerbated RV mitochondrial dysfunction in response to acute $\mathrm{O}_{2}$ deprivation is directly related to an inhibition of respiratory chain, because the difference was detected in uncoupled post-anoxic respiration which does not depend upon activity of adenine-nucleotide translocator 1 (Figure 3e). Finally, electron microscopy revealed that acute oxygen deprivation markedly affected RV-mitochondrial morphology to a similar extent in both groups of rats. Yet, functional outcome was significantly worse only in organelles isolated from IUGR-born animals. This suggests that IUGR affects only functional response of RV mitochondria to acute hypoxic stress.

We have shown that when exposed to the acute hypoxia, the RV in the IUGR-born adult male rats does not function as efficiently as that of controls, and their RV mitochondrial function is significantly poorer compared with that in adult rats normally nourished at birth. Thus, in the adulthood, fetal IUGR selectively predispose RV mitochondria to a more severe inhibition in response to acute hypoxic stress. This highlights mitochondria as a novel mechanistic target for IUGR-associated maladaptive RV response to hypoxic stress.

\section{METHODS}

All chemicals were supplied by Sigma-Aldrich (St. Louis, MO) except for $\mathrm{N}$-2-hydroxyethylpiperazine-N9-2-ethanesufonic acid (HEPES) by Research Organics (Cleveland, $\mathrm{OH}$ ).

\section{Animals and Experimental Design}

The experimental protocol was approved by the Institutional Animal Care and Use Committee of Columbia University in accordance with Association for Assessment and Accreditation of Laboratory 


\section{Articles $\quad$ Keenaghan et al.}

Animal Care. Timed-pregnant Sprague-Dawley rats (Charles River Laboratories, Stone Ridge, NY) at gestational day 14 (GD14) were randomly divided into two groups, control and nutrient restriction. All rats were housed individually in standard rat cages with free access to water. The weight was recorded daily throughout pregnancy. Control rats were fed a standard rat chow ad libitum. In the nutrient restriction group food intake was limited to $40 \%(11.5 \pm 0.2 \mathrm{~g} / \mathrm{d})$ (3.4 kcal/g, fat $13 \%$, protein $27 \%$, carbohydrate $60 \%$; LabDiet, St. Louis, MO) of the average maternal standard rat chow until delivery $(20,21)$. On postnatal day 1 ( 1$)$, undernourished dams were fed ad libitum for $1 \mathrm{~d}$ and resumed the restricted diet on $\mathrm{p} 2$ until p7. On $\mathrm{p} 1$ all litters were culled to six pups per dam. Pups were weighed daily for the first $7 \mathrm{~d}$ of life and then weekly until p90. Pups born to undernourished dams were assigned to IUGR group and pups born to rats fed ad libitum served as control. Following weaning from maternal milk all pups were housed two or three per cage with food access ad libitum.

At p90-120 offspring rats from both groups were exposed to hypoxic challenge $\left(8 \% \mathrm{O}_{2}\right.$ balanced $\left.\mathrm{N}_{2}\right)$ for $15 \mathrm{~min}$ at room temperature. Hypoxia exposure was performed under isoflurane (2.5 vol\% induction, 1.7 vol\% maintenance) anesthesia. During hypoxic challenge oxygen saturation $\left(\mathrm{SaO}_{2}\right), \mathrm{HR}, \mathrm{RR}$, and cardiac function $(\mathrm{ECHO})$ were monitored. Two IUGR-born rats died during the hypoxia trials. One death occurred within $10 \mathrm{~min}$ and another was at $15 \mathrm{~min}$ of $8 \%$ oxygen exposure. Data from these animals were excluded from analysis.

$\mathrm{SaO}_{2}, H R$, and $R R$ were measured using MouseOx, a Small Animal Pulse Oximeter (Starr Life Sciences, Oakmont, PA). Briefly, an infrared sensor clip was placed on the left rear paw for recording. After stable recording of $5 \mathrm{~min}$, the ambient air was replaced by $8 \%$ oxygen (Matheson Tri-Gas, Basking Ridge, NJ) for $15 \mathrm{~min}$. All abovementioned vital signs were monitored and recorded using MouseOx Software continuously during hypoxic exposure and for $3 \mathrm{~min}$ of reoxygenation. For data analysis we selected values with interval of $1 \mathrm{~min}$ prior to, during, and after hypoxic challenge.

ECHO was performed with a Sonos 5500 (Philips Medical Systems, Andover, MA) using a dynamically focused $12 \mathrm{MHz}$ transducer (S12). $\mathrm{B}$-mode and M-mode two-dimensional ECHO images were obtained in parasternal long- and short-axis views. In the B-mode short-axis images, LV and RV endocardial area tracings were performed on-line at the mid of papillary muscle level at end diastole. Dimensions of LV and RV diameter were measured using an M-mode left parasternal short-axis view at mid papillary level. Tricuspid annular plane systolic excursion, which expresses the motion of tricuspid annulus and represents a parameter for RV systolic function, was also recorded in the M-mode from apical four-chamber view. After baseline (prior to hypoxic stress) ECHO assessment, an echocardiogram was repeated at $8 \mathrm{~min}$ of hypoxia, once physiological response to hypoxia (changes in the $\mathrm{HR}$ and $\mathrm{RR}$ and $\mathrm{SaO}_{2}$ ) reached a steady state. At 2 min after reoxygenation with room air, ECHO was repeated again and considered as post-hypoxic study. All measurements were averaged at least over three cardiac cycles. An experienced operator, blinded to the study groups, preformed and analyzed ECHO data.

\section{Isolation of Mitochondria}

Total cardiac mitochondria were isolated as described by TokarskaSchlattner (37) with modifications (38). Briefly, at the age of full sexual and cardiovascular systems maturation p120 through p180 (39), animals from both groups were killed and the heart was quickly excised, RV and LV wall was removed separately, washed and cut into pieces in ice-cold isolation buffer ( $225 \mathrm{mmol} / \mathrm{l}$ mannitol, $75 \mathrm{mmol} / \mathrm{l}$ sucrose, $1 \mathrm{mmol} / \mathrm{l}$ ethyleneglycol-bis(baminoethylether)-N,N9-tetraacetic acid (EGTA), 5 $\mathrm{mmol} / \mathrm{l} \mathrm{HEPES}, 1 \mathrm{mg} / \mathrm{ml}$ of fatty acid free bovine serum albumin (BSA), $\mathrm{pH}$ 7.4), and homogenized using a Glass-glass homogenizer (Kontes Dounce Tissue Grinder, Kimble" catalog number K8853000007) with a looser pestle for 10 strokes then a tighter pestle for 5 strokes in $5 \mathrm{ml}$ isolation buffer. Nuclei and cell debris were removed by centrifugation at $1,100 \mathrm{~g}$ for $2 \mathrm{~min}$ at $4^{\circ} \mathrm{C}$. The resulting supernatant containing crude mitochondrial pellet was further purified by 15 -min centrifugation at $18,500 \mathrm{~g}$ on a $15 \%$ Percoll gradient. Percoll was removed from the purified mitochondrial fraction by washing the pellet in sucrose buffer (250 mmol/l sucrose, $1 \mathrm{mmol} / \mathrm{l} \mathrm{EGTA,} 5 \mathrm{mmol} / \mathrm{l} \mathrm{HEPES,} 1 \mathrm{mg} / \mathrm{ml}$ of
BSA, pH 7.4) at $10,000 \mathrm{~g}$ for $10 \mathrm{~min}$. Finally, the pellet was resuspended in the albumin-free sucrose buffer and kept on ice for further analysis. Mitochondrial protein concentration was determined by the Bradford method using the Bio-Rad Protein Assay with BSA as a standard.

Mitochondrial respiration was measured using an Oxytherm respirometer (Hansatech Instrument, England). Respiratory buffer consisted of $200 \mathrm{mmol} / \mathrm{l}$ sucrose, $25 \mathrm{mmol} / \mathrm{l} \mathrm{KCl,} 2 \mathrm{mmol} / \mathrm{K} \mathrm{KH}_{2} \mathrm{PO}_{4}$, $5 \mathrm{mmol} / \mathrm{l} \mathrm{MgCl}, 5 \mathrm{mmol} / \mathrm{l}$ HEPES, $0.03 \mathrm{mmol} / \mathrm{l}$ Ap5A (P1,P5Diadenosine- $5^{\prime}$ pentaphosphate pentasodium salt), and $0.2 \mathrm{mg} / \mathrm{ml}$ BSA and adjusted to $\mathrm{pH} 7.2$ with $\mathrm{KOH}$. Total $0.1 \mathrm{mg}$ of mitochondrial protein per milliliter buffer was used. Mitochondrial resting respiration rates were measured using either malate $10 \mathrm{mmol} / \mathrm{l} /$ glutamate $5 \mathrm{mmol} / \mathrm{l}$ or succinate $5 \mathrm{mmol} / \mathrm{l} /$ glutamate $5 \mathrm{mmol} / \mathrm{l}$ as substrates. Following recording the resting respiration rate (state 4), phosphorylating respiration (state 3 ) was initiated by adding $100 \mathrm{nmol} / \mathrm{l}$ ADP. State 3 refers to oxygen consumption in the presence of ADP, and state 4 is the rate of oxygen consumption after phosphorylation of all available ADP. Once ADP phosphorylation was completed, mitochondrial state 4 respiration was recorded again for the same amount of time that was needed to complete state 3 respiration. To initiate an uncoupling of respiration, $70 \mathrm{nmol} / \mathrm{l}$ of DNP was added to the reaction mixture. The time point when mitochondria consumed all oxygen in the buffer was recorded as the beginning of anoxia. Following $30 \mathrm{~min}$ of complete anoxia, the chamber was opened and when oxygen content in the buffer returned to the level at which DNP was added, the chamber was closed and the rate of $\mathrm{O}_{2}$ consumption recorded. The changes in the DNP-accelerated $\mathrm{O}_{2}$-consumption rate after anoxic challenge (DNP2) were expressed in the percentage of the DNP-accelerated rate recorded prior to anoxia (DNP1). This value was used to define the extent of post-anoxic inhibition of the electron transport chain. Thus, the ratio of DNP2 (post-anoxia rate) to DNP1 (pre-anoxia rate) represents the ability of mitochondrial respiratory chain to recover after anoxia.

In randomly selected animals from both groups, mitochondrial pellets (control and post-anoxia) were processed for electron microscopy to assess mitochondrial morphology. In brief, mitochondrial pellets were fixed overnight in $2.5 \%$ glutaraldehyde and $4 \%$ paraformaldehyde in $0.1 \mathrm{M}$ cacodylate buffer $(\mathrm{pH} 7.4)$ at $4^{\circ} \mathrm{C}$. Then they were post-fixed with $1 \%$ osmium tetroxide in $0.1 \mathrm{M}$ cacodylate buffer, dehydrated, and embedded in Epon-Araldite. Ultrathin sections were cut using a Reichert Ultramicrotome, stained with uranyl acetate and lead citrate, and examined under a JEOL 1200 electron microscope (JEOL LTD, Japan).

Statistical analyses were performed using unpaired two-tailed Student's $t$-tests to detect differences between control and IUGR groups. To analyze changes in the same indexes over the time, twoway ANOVA for repeated measures with Fisher's post-hoc analysis of summated values were used. Data are presented as means $\pm \mathrm{SE}<0.05$ was considered significant. Data analyzed with GraphPad Prism 5.0 for Windows (GraphPad Software, San Diego, CA). For all graphs, the bars represent the means $\pm \mathrm{SE}$.

\section{STATEMENT OF FINANCIAL SUPPORT}

No financial support.

Disclosure: The authors have no financial ties to products in the study or other conflicts of interest.

\section{REFERENCES}

1. Hales CN, Barker DJ. Type 2 (non-insulin-dependent) diabetes mellitus: the thrifty phenotype hypothesis. Diabetologia 1992;35:595-601.

2. Gluckman PD, Hanson MA. Developmental origins of disease paradigm: a mechanistic and evolutionary perspective. Pediatr Res 2004;56:311-7.

3. Hanson M, Gluckman P, Bier D, et al. Report on the $2^{\text {nd }}$ World Congress on Fetal Origins of Adult Disease, Brighton, U.K., June 7-10, 2003. Pediatr Res 2004;55:894-7.

4. Gardner DS, Hosking J, Metcalf BS, Jeffery AN, Voss LD, Wilkin TJ. Contribution of early weight gain to childhood overweight and metabolic health: a longitudinal study (EarlyBird 36). Pediatrics 2009;123:e67-73.

5. Sébert SP, Hyatt MA, Chan LL, et al. Influence of prenatal nutrition and obesity on tissue specific fat mass and obesity-associated (FTO) gene expression. Reproduction 2010;139:265-74. 
6. Unterberger A, Szyf M, Nathanielsz PW, Cox LA. Organ and gestational age effects of maternal nutrient restriction on global methylation in fetal baboons. J Med Primatol 2009;38:219-27.

7. Rizzo G, Arduini D, Romanini C. Doppler echocardiographic assessment of fetal cardiac function. Ultrasound Obstet Gynecol 1992;2:434-45.

8. Verburg BO, Jaddoe VW, Wladimiroff JW, Hofman A, Witteman JC, Steegers EA. Fetal hemodynamic adaptive changes related to intrauterine growth: the Generation R Study. Circulation 2008;117:649-59.

9. Sebert S, Sharkey D, Budge H, Symonds ME. The early programming of metabolic health: is epigenetic setting the missing link? Am J Clin Nutr 2011;94:Suppl 6:1953S-8S.

10. Corstius HB, Zimanyi MA, Maka N, et al. Effect of intrauterine growth restriction on the number of cardiomyocytes in rat hearts. Pediatr Res 2005;57:796-800.

11. Welder AA, Grant R, Kutschke RL, Anthony M, Bradlaw J, Acosta D. Effects of maternal calorie-restricted diet on development of the foetal heart, as evaluated in primary cultures of rat myocardial cells. Food Chem Toxicol 1991;29:445-52.

12. Colom B, Oliver J, Roca P, Garcia-Palmer FJ. Caloric restriction and gender modulate cardiac muscle mitochondrial $\mathrm{H}_{2} \mathrm{O}_{2}$ production and oxidative damage. Cardiovasc Res 2007;74:456-65.

13. Gibson K, Halliday JL, Kirby DM, Yaplito-Lee J, Thorburn DR, Boneh A. Mitochondrial oxidative phosphorylation disorders presenting in neonates: clinical manifestations and enzymatic and molecular diagnoses. Pediatrics 2008;122:1003-8.

14. Peterside IE, Selak MA, Simmons RA. Impaired oxidative phosphorylation in hepatic mitochondria in growth-retarded rats. Am J Physiol Endocrinol Metab 2003;285:E1258-66.

15. Selak MA, Storey BT, Peterside I, Simmons RA. Impaired oxidative phosphorylation in skeletal muscle of intrauterine growth-retarded rats. Am J Physiol Endocrinol Metab 2003;285:E130-7.

16. Griffiths ER, Friehs I, Scherr E, Poutias D, McGowan FX, Del Nido PJ. Electron transport chain dysfunction in neonatal pressure-overload hypertrophy precedes cardiomyocyte apoptosis independent of oxidative stress. J Thorac Cardiovasc Surg 2010;139:1609-17.

17. Nouette-Gaulain K, Malgat M, Rocher C, et al. Time course of differential mitochondrial energy metabolism adaptation to chronic hypoxia in right and left ventricles. Cardiovasc Res 2005;66:132-40.

18. Redout EM, Wagner MJ, Zuidwijk MJ, et al. Right-ventricular failure is associated with increased mitochondrial complex II activity and production of reactive oxygen species. Cardiovasc Res 2007;75:770-81.

19. Vuguin PM. Animal models for small for gestational age and fetal programming of adult disease. Horm Res 2007;68:113-23.

20. Williams SJ, Campbell ME, McMillen IC, Davidge ST. Differential effects of maternal hypoxia or nutrient restriction on carotid and femoral vascular function in neonatal rats. Am J Physiol Regul Integr Comp Physiol 2005;288:R360-7.

21. Xu Y, Williams SJ, O'Brien D, Davidge ST. Hypoxia or nutrient restriction during pregnancy in rats leads to progressive cardiac remodeling and impairs postischemic recovery in adult male offspring. FASEB J 2006;20:1251-3.

22. Garofano A, Czernichow P, Bréant B. Postnatal somatic growth and insulin contents in moderate or severe intrauterine growth retardation in the rat. Biol Neonate 1998;73:89-98.

23. Martin-Gronert MS, Ozanne SE. Experimental IUGR and later diabetes. J Intern Med 2007;261:437-52.
24. Battista MC, Oligny LL, St-Louis J, Brochu M. Intrauterine growth restriction in rats is associated with hypertension and renal dysfunction in adulthood. Am J Physiol Endocrinol Metab 2002;283:E124-31.

25. Woodall SM, Breier BH, Johnston BM, Gluckman PD. A model of intrauterine growth retardation caused by chronic maternal undernutrition in the rat: effects on the somatotrophic axis and postnatal growth. J Endocrinol 1996;150:231-42.

26. Menendez-Castro C, Fahlbusch F, Cordasic N, et al. Early and late postnatal myocardial and vascular changes in a protein restriction rat model of intrauterine growth restriction. PLoS One 2011;6:e20369.

27. Blondeau B, Garofano A, Czernichow P, Bréant B. Age-dependent inability of the endocrine pancreas to adapt to pregnancy: a longterm consequence of perinatal malnutrition in the rat. Endocrinology 1999;140:4208-13.

28. Komniski MS, Yakushev S, Bogdanov N, Gassmann M, Bogdanova A. Interventricular heterogeneity in rat heart responses to hypoxia: the tuning of glucose metabolism, ion gradients, and function. Am J Physiol Heart Circ Physiol 2011;300:H1645-52.

29. Galkin A, Abramov AY, Frakich N, Duchen MR, Moncada S. Lack of oxygen deactivates mitochondrial complex I: implications for ischemic injury? J Biol Chem 2009;284:36055-61.

30. Vinogradov AD. Catalytic properties of the mitochondrial NADH-ubiquinone oxidoreductase (complex I) and the pseudo-reversible active/inactive enzyme transition. Biochim Biophys Acta 1998;1364:169-85.

31. Brown GC, Borutaite V. Inhibition of mitochondrial respiratory complex I by nitric oxide, peroxynitrite and S-nitrosothiols. Biochim Biophys Acta 2004;1658:44-9.

32. Loskovich MV, Grivennikova VG, Cecchini G, Vinogradov AD. Inhibitory effect of palmitate on the mitochondrial NADH:ubiquinone oxidoreductase (complex I) as related to the active-de-active enzyme transition. Biochem J 2005;387(Pt 3):677-83.

33. Chen Q, Moghaddas S, Hoppel CL, Lesnefsky EJ. Reversible blockade of electron transport during ischemia protects mitochondria and decreases myocardial injury following reperfusion. J Pharmacol Exp Ther 2006;319:1405-12.

34. Lesnefsky EJ, He D, Moghaddas S, Hoppel CL. Reversal of mitochondrial defects before ischemia protects the aged heart. FASEB J 2006;20:1543-5.

35. Niatsetskaya ZV, Sosunov SA, Matsiukevich D, et al. The oxygen free radicals originating from mitochondrial complex I contribute to oxidative brain injury following hypoxia-ischemia in neonatal mice. J Neurosci 2012;32:3235-44.

36. Lane RH, Chandorkar AK, Flozak AS, Simmons RA. Intrauterine growth retardation alters mitochondrial gene expression and function in fetal and juvenile rat skeletal muscle. Pediatr Res 1998;43:563-70.

37. Tokarska-Schlattner M, Dolder M, Gerber I, Speer O, Wallimann T, Schlattner U. Reduced creatine-stimulated respiration in doxorubicin challenged mitochondria: particular sensitivity of the heart. Biochim Biophys Acta 2007;1767:1276-84.

38. Caspersen CS, Sosunov A, Utkina-Sosunova I, Ratner VI, Starkov AA, Ten VS. An isolation method for assessment of brain mitochondria function in neonatal mice with hypoxic-ischemic brain injury. Dev Neurosci 2008;30:319-24.

39. Fischer A, Ten Hove M, Sebag-Montefiore L, et al. Changes in creatine transporter function during cardiac maturation in the rat. BMC Dev Biol 2010;10:70. 